Proyecciones

Vol. 15, No 2, pp. 169-177, December 1996

Universidad Católica del Norte

Antofagasta - Chile

\title{
SUR L'HOMOLOGIE ELLIPTIQUE DU SPECTRE DE MILNOR
}

\author{
Abdelaziz Kheldouni and Fayçal Lamrini. \\ Université Sidi Mohammed Benabdallah, Marruecos
}

\begin{abstract}
The main purpose of this paper is to give a splitting result of the Hopf algebra $E l l^{*}(E l l)$ for the elliptic spectrum Ell : $\mathcal{E} l l^{*}(E l l)=$ $H o m_{\Omega^{U}}^{*}\left(M U_{*}(E l l), A\right) \oplus E_{x} t_{\Omega_{*}^{L}}^{1, *}\left(M U_{*}(E l l), A\right)$, where $A=E l l_{*}(p t)$.
\end{abstract}




\section{Introduction}

Dans ([4], 9) P. E. Conner et L. Smith ont étudié, en se basant sur les résolutions de U-bordisme d'un CW-complexe fini $\mathrm{X}$, l'éxistence d'une suite spectrale $\left(E_{r}[X], d_{r}[X]\right)$ qui converge vers $K^{*}(X)$ et de deuxième terme $E_{2}^{p, q}[X]=\operatorname{Ext}_{\Omega^{U}}^{p, q}\left(M U_{*}(X), \mathbf{Z}\right)$. Z étant muni de sa structure de $\Omega_{*}^{U}$-module donnée par le genre de Todd. De plus en remarquant que $\operatorname{Ext}_{\Omega_{U}^{U}}^{p, q}\left(M U_{*}(X), \mathbf{Z}\right)=\mathbf{0}$ pour $p>1$, ils ont déduit une suite éxacte scindée (cf. [4], Proposition 9.8):

$$
0 \rightarrow \operatorname{Ext}_{\Omega_{\star}^{U}}^{1, n-1}\left(M U_{*}(X), Z\right) \rightarrow K^{n}(X) \rightarrow \operatorname{Hom}_{\Omega_{*}^{U}}^{-n}\left(M U_{*}(X), Z\right) \rightarrow 0(1)
$$

qui permet d'identifier le sous-groupe de torsion de $K^{*}(X)$ à $\operatorname{Ext}_{\Omega^{U}}^{1, *}\left(M U_{*}(X), \mathbf{Z}\right)$. D'autre part, pour une théorie colomologique représentée par un $M U$-spectre module $E$, un théorème d'Adams ([1], Theorem 13.6), donne une suite spectrale qui converge vers $E^{*}(X)$ et de deuxiéme terme $\operatorname{Ext}_{\Omega_{*}^{U}}^{p, q}\left(M U_{*}(X), \pi_{*}(E)\right)$. La proposition 9.8 de [4] devient une conséquence immédiate du théorème d'Adams. Ceci suggère une généralisation des résultats de $([4], 9)$ à une théorie cohomologique définie par un groupe formel; travail qui fait l'objet de ce papier. Nous montrons les résultats suivants:

Proposition 3.2.

$E l l_{*}(M U)$ est un $\Omega_{*}^{U}$-module plat

Théorème 3.3.

$$
\operatorname{Dimhom}_{\Omega_{*}^{U}}\left(-\mathcal{E} l l^{*}(M U)\right) \leq 1 .
$$

\section{Préliminaires}

La théorie cohomologique $M U^{*}(-)$ associée au spectre de Milnor $M U$ est une théorie multiplicative $\mathbf{C}$-orientée, universelle pour les cohomologies multiplicatives $\mathbf{C}$-orientées définies sur la catégorie stable de Boardman. Son groupe formel $F^{U}(X, Y)$ induit par la $\mathbf{C}$-orientation est le groupe 
formel universel dont le logarithme est donné par la formule de Miscenko [1] :

$$
g_{U}(X)=X+\sum_{n \grave{o} 1} \frac{\left[\mathbf{C} P^{n}\right]}{n+1} X^{n+1} \in \Omega_{*}^{U} \otimes \mathbf{Q}[[\mathbf{X}]] .
$$

Soit $R$ un anneau gradué muni d'un groupe formel $F(X, Y)$. $F$ est classifié par un genre $\varphi: \Omega_{*}^{U} \rightarrow R$ (i.e. $\varphi$ est un homomorphisme d'anneaux tel que $\varphi_{*} F^{U}=F$ ). On munit alors $R$ d'une structure de $\Omega_{*}^{U}$-module via $\varphi$, et on considère le foncteur dfini sur la catégorie stable de Boardman

$$
\mathcal{E}^{*}: X \rightarrow M U^{*}(X) \otimes_{\Omega_{\Xi}^{U}} R=\mathcal{E}^{*}(X) .
$$

Pour tout entier naturel n, la n-série associée au groupe formel $F(X, Y)$ est définie par récurrence par

$[1]_{F}(X)=X$

$[\mathrm{n}]_{F}(X)=F\left(X,[n-1]_{F}(X)\right), \forall n \in \mathbf{N}$.

Si p est un nombre premier, on écrit

$[p]_{F}(X)=p X+\ldots+a_{p, 1} X^{p}+\ldots+a_{p, k} X^{p^{k}}+\ldots$

Théorème 1.1 ([5]): Le foncteur $\mathcal{E}^{*}(X)=M U^{*}(X) \otimes_{\Omega_{*}^{U}} R$ définit une théorie cohomologique, si pour tout nombre premier $p$, la suite $\left(p, a_{p, i}\right)_{i \geq 1}$ est régulière dans $R$.

En fait, $\mathcal{E}^{*}(X)$ est une théorie cohomologique multiplicative $\mathbf{C}$-orientée (voir [6]). Elle est donc représentée par un spectre-anneau $\mathbf{E}$ dont les applications de structure seront notées $\mu_{\mathbf{E}}: \mathbf{E} \wedge \mathbf{E} \rightarrow \mathbf{E}$ et $\iota_{\mathbf{E}}: \mathbf{S} \rightarrow \mathbf{E}$. Notons que La structure de spectre anneau sur $\mathbf{E}$, est celle qui induit la transformation naturelle $\mathcal{E}^{*}(X) \otimes \mathcal{E}^{*}(X) \rightarrow \mathcal{E}^{*}(X)$ obtenue grâce à la structure multiplicative de $M U^{*}(-)$. De plus, en vertu de l'universalité du cobordisme complexe, il existe une application de spectres $\widetilde{\phi}: M U \rightarrow \mathbf{E}$, définissant la transformation naturelle $\phi: M U^{*}(-) \rightarrow \mathcal{E}^{*}(-)$ qui classifie $\mathcal{E}^{*}(X)=M U^{*}(X) \otimes_{\Omega_{*}} R$, et telle que $\widetilde{\phi}_{*}(p t)=\varphi$.

La composition $\nu: M U \wedge E \stackrel{\widetilde{\phi} \wedge 1_{E}}{\longrightarrow} E \wedge E \stackrel{\mu}{\longrightarrow} E$, confère à $\mathbf{E}$ une structure de $M U$-spectre module. Là aussi, on remarque que la transformation $\nu_{*}$ : $(M U \wedge E)_{*}(X) \rightarrow E_{*}(X)$ induite par $\nu$ peut être obtenue par la composition suivante

$(M U \wedge \mathbf{E})_{*}(X) \simeq(\mathbf{E} \wedge M U)_{*}(X) \simeq \mathbf{E}_{*}(M U \wedge X)=$

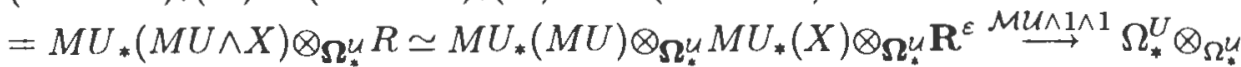
$M U_{*}(X) \otimes_{\Omega_{*}^{u}} R \simeq M U^{*}(X) \otimes_{\Omega_{*}^{u}} R=\mathbf{E}_{*}(X) \cdot \varepsilon_{M U}: M U_{*}(M U) \rightarrow \Omega_{*}^{U}$ est l'augmentation de l'algèbroïde de $\operatorname{Hopf} M U_{*}(M U)$. 


\section{Sur le théorème du coefficient universel}

Soit $E$ un spectre-anneau et $F$ un spectre-module sur $E$, avec comme applications structurales $\iota: \mathbf{S} \longrightarrow E, \mu: \mathbf{E} \wedge \longrightarrow E$. Pour tout spectre $X$ et tout $\alpha \in F^{n}(X)$ nous avons un homomorphisme de $\pi_{*}(E)$-modules $\zeta_{X}(\alpha): E(X) \rightarrow \pi_{*}(F)$ défini de la manière suivante:

étant donnée une application $f: X \rightarrow \Sigma^{n} F$ représentant $\alpha$, alors pour tout $u \in E_{k}(X)$, on pose $\zeta_{X}(\alpha)(u)=\nu_{*} o f_{*}(u) \in \pi_{k-n}(F)$. Nous avons ainsi une transformation naturelle :

$$
\zeta_{X}: F^{n}(X) \rightarrow \operatorname{Hom}_{\pi \cdot(E)}^{-n}\left(E_{*}(X) ; \pi_{*}(F)\right)
$$

Les spectres $E$ qui seront utilisés dans ce paragraphe vrifient la condition (C) suivante (voir [1], 13.6) :
$\left\{\begin{array}{l}\mathrm{E} \text { est limite inductive d'une famille }\left\{E_{\alpha}\right\} \text { de sous-spectres finis } \\ E_{\alpha} \text { tels que : }\end{array}\right.$
(C) $\left\{\right.$ i) $E_{*}\left(D E_{\alpha}\right)$ est un $\pi_{*}\left(E_{\alpha}\right)$-module projectif.
ii) $D E_{\alpha}$ est un spectre pour lequel $\zeta_{D E_{\alpha}}$ est un isomorphisme, où $D E_{\alpha}$ désigne le $S$-dual de $E_{\alpha}$.

L'interrêt de cette condition réside dans le fait qu'elle permet de fabriquer des résolutions du spectre $X$ qui donnent la suite spectrale d'AdamsNovikov. Par exemple les spectres $S, H Z_{p}, M U, M O, M S p, K, K O$ vérifient la condition (C). Il est bien connu (cf. [1], Theorem 13.6) que si $E$ est un spectre anneau vérifiant $(\mathbf{C})$, et $F$ un spectre module sur $E$, alors pour tout spectre $X$, il xiste une suite spectrale qui converge vers $F^{*}(X)$, de deuxième terme $E_{2}^{p, q}=\operatorname{Ext}_{\pi_{*}(E)}^{p, q}\left(E_{*}(X),: \pi_{*}(F)\right)$ et dont le "edge-homomorphisme" coïncide avec la transformation (2). En particulier, en prenant pour $E$ le spectre $M U$, et pour $F$ le spectre $\mathbf{E}$ représentant la théorie cohomologique $\mathcal{E}^{*}(X)=M U^{*}(X) \otimes_{\Omega^{U}} R$ définie dans 1 , nous avons une suite spectrale $\tilde{E}_{r}^{p, q}$ qui converge vers $\mathcal{E}^{*}(X)$ de deuxime terme $\tilde{E}_{2}^{p, q}=\operatorname{Ext}_{\Omega_{*}^{U}}^{p, q}\left(E_{*}(X), R\right)$ et dont le "edge-homomorphisme" est la transformation

$$
\zeta_{X}: \mathcal{E}^{*}(X) \longrightarrow \operatorname{Hom}_{\Omega_{*}^{U}}\left(M U_{*}(X), R\right)
$$

Comme conséquence immédiate on a :

Corollaire 2.1: Si $X$ est un spectre tel que $M U_{*}(X)$ est un $\Omega_{*}^{U}$-module projectif, alors

$$
\zeta_{X}: \mathcal{E}^{*}(X) \longrightarrow \operatorname{Hom}_{\Omega_{*} U}\left(M U_{*}(X), R\right)
$$

est un isomorphisme. 
Dans le cas où $X$ est un CW-complexe fini, on peut étendre la proposition 9.5 de [4] à la théorie cohomologique $\mathcal{E}^{*}(X)=M U^{*}(X) \otimes_{\Omega_{*}^{U}} R$. Nous avons alors.

Lemme 2.2: Pour tout $p>0$ et tout CW-complxe fini $X$, on a

$$
\operatorname{Tor}_{p, *}^{\Omega_{*}^{U}}\left(M U_{*}(X), R\right)=0 .
$$

Démonstration: Il suffit de reprendre la démonstration de ([4], Proposition 9.5) qui s'adapte bien à notre situation.

Nous dduisons de ([2], chap. VI, 4.1.3) et du lemme 2.2.

Proposition 2.3: Pour tout CW-complexe fini $X$, nous avons un isomorphisme :

$$
\operatorname{Ext}_{R}^{n, *}\left(E_{*}(X), R\right) \rightarrow \operatorname{Ext}_{\Omega_{*}^{U, *}}^{n, *}\left(M U_{*}(X), R\right) .
$$

En appliquant ces résultats à la $\mathrm{K}$-théorie, nous retrouvons les conclusions de ([4], 9). En effet, $K^{*}(X)$ est une théorie cohomologique $\mathbf{C}$-orientée dont l'anneau de base $K^{*}(p t)$ est $\mathbf{Z}\left[u, u^{-1}\right]$ avec $u$ le générateur de $K^{-2}(p t)$ (voir [1]). Son spectre $B U$ est classifiépar une application $\theta: M U \rightarrow K$ qui induit le genre de Todd, $T d: \Omega_{*}^{U} \rightarrow \mathbf{Z}\left[u, u^{-1}\right]$. De plus nous avons $K^{*}(X)=M U^{*}(X) \otimes_{\Omega_{*}^{U}} Z\left[u, u^{-1}\right]$ (voir [3]), ou $Z\left[u, u^{-1}\right]$ est un $\Omega_{*}^{U}$-module grâce à Td. En vertu de la proposition 2.3 on obtient l'isomorphisme $\operatorname{Ext}_{\mathbf{Z}\left[u, u^{-1}\right]}^{n, *}\left(K_{*}(X), Z\left[u, u^{-1}\right\}\right) \simeq \operatorname{Ext}_{\Omega_{*}^{U}}^{n, *}\left(M U_{*}(X), Z\left[u, u^{-1}\right]\right)$. De plus puisque $\mathbf{Z}\left[u, u^{-1}\right]$ est un $\mathbf{Z}$-module libre, $\operatorname{Tor}_{\mathbf{Z}}^{n, *}\left(-, Z\left[u, u^{-1}\right]\right)=\mathbf{0}$, donc d'après $\quad\left([2]\right.$, chap. VI, 4.1.3), $\quad \operatorname{Ext}_{\left.\mathbf{Z} \mid u, u^{-1}\right\}}^{n, *}\left(-, Z\left[u, u^{-1}\right]\right)=$ $\operatorname{Ext}_{Z}^{n, *}\left(-, Z\left[u, u^{-1}\right]\right)=0, \forall n>1$ car $Z$ est un anneau principal. Donc pour tout CW-complexe fini $X$ et tout $n>1, \operatorname{Ext}_{\mathbf{Z}\left[u, u^{-1}\right]}^{n, *}\left(K_{*}(X), Z\left[u, u^{-1}\right]\right)=0$, d'où $\operatorname{Ext}_{\Omega^{U}}^{n, *}\left(M U_{*}(X), Z\left[u, u^{-1}\right]\right)=0$. La suite spectrale d'Adams-Novikov est donc triviale pour $p$ différent de 0 et de 1; et d'après ([2], chap. XV 5) nous avons une suite exacte scindée:

$$
0 \rightarrow E_{2}^{1, n-1} \rightarrow K^{n}(X) \rightarrow E_{2}^{0, n} \rightarrow 0
$$

ou encore

$$
\begin{aligned}
0 \rightarrow \operatorname{Ext}_{\Omega^{U}}^{1, n-1}\left(M U_{*}(X), \mathbf{Z}\left[u, u^{-1}\right]\right) & \rightarrow K^{n}(X) \rightarrow H_{\Omega_{*}^{U}}^{-n}\left(M U_{*}(X),\right. \\
\left.\mathbf{Z}\left[u, u^{-1}\right]\right) & \rightarrow 0
\end{aligned}
$$

on retrouve alors le théorème 9.8 de [4]. 


\section{Cas de la cohomologie elliptique}

Le genre elliptique est un homomorphisme d'anneaux $f: M U_{*} \rightarrow A$ où $A$ est une Q-algèbre, défini par son logarithme $g$ qui est une série formelle donnée par l'intégrale elliptique de première espèce,

$$
g(X)=\int_{0}^{X} \frac{d t}{\sqrt{1-2 \delta t^{2}+\varepsilon t^{4}}}
$$

où $\delta, \varepsilon \in A$. On constate facilement que $g(X)$ est à coefficients dans $\mathbf{Z}\left[\frac{1}{2}\right][\delta, \varepsilon]$. Si $\delta$ et $\varepsilon$ sont $\mathbf{Q}$-algèbriquement indépendants, le théorème du foncteur exact de Landweber est vérifié (cf. [6]). En localisant l'anneau $\mathbf{Z}\left[\frac{1}{2}\right][\delta, \varepsilon]$ en $\gamma=\varepsilon, \delta^{2}-\varepsilon$ ou $\Delta=\varepsilon\left(\delta^{2}-\varepsilon\right)^{2}$, nous obtenons une théorie cohomologique C-orientée $\mathcal{E}^{*}(X)=M U^{*}(X) \otimes_{\Omega_{*}^{U}} \mathbf{Z}\left[\frac{1}{2}\right]\left[\delta, \varepsilon, \gamma^{-1}\right]$, appelée cohomologie elliptique (cf. [6], [7]). L'anneau de base $\mathcal{A}_{*}=\mathbf{Z}\left[\frac{1}{2}\right]\left[\delta, \varepsilon, \gamma^{-1}\right]$ s'identifie à l'anneau de formes nodulaires pour le groupe $\Gamma_{0}(2)=\left\{\left(\begin{array}{cc}a & b \\ 2 c & d\end{array}\right) \in S L_{2}(\mathbf{Z})\right\}$ dont les coefficients de la q-série appartiennent $\mathbf{Z}\left[\frac{1}{2}\right]$ ayant des pôles à l'infini, en o ou aux deux cusp (respectivement). D'aprés §.1, la cohomologie $\mathcal{E}^{*}(X)=M U^{*}(X) \otimes_{\Omega_{*}^{U}} \mathcal{A}_{*}$ est représentée par un spectre-anneau $E l l$ qui est un spectre-module sur $M U$, et sera note $\mathcal{E} l l^{*}(X)$. L aussi nous avons une suite spectrale d'Adams-Novikov pour tout spectre $\mathrm{X}$ :

$$
E_{2}^{p, *}=\operatorname{Ext}_{\Omega_{*}^{U}}^{p, *}\left(M U_{*}(X), \mathcal{A}_{*}\right) \Rightarrow E l l^{*}(X)
$$

Le "edge-homomorphisme" coïncide avec la transformation (2) du $\S .2$ :

$$
\zeta_{X}: E l l^{*}(X) \rightarrow \operatorname{Hom}_{\Omega^{U}}^{*}\left(M U_{*}(X), \mathcal{A}_{*}\right)
$$

Nous utiliserons le lemme suivant, dont la démonstration est analogue à celle donnée dans [9], ơl'on remplace $\mathrm{BP}$ par $\mathrm{MU}$ :

Lemme 3.1. Tout $M U_{*}(M U)$-comodule est linite inductive filtrée d'une famille de $M U_{*}(M U)$-comodules de prsentation finie en tant que $\Omega_{*}^{U}$-modules.

Proposition 3.2. $E l l^{*}(M U)$ est un $\Omega_{*}^{U}$-module plat. 
Démonstration: En utilisant l'unité gauche $\eta_{L}: \Omega_{*}^{U} \rightarrow M U_{*}(M U)$ et la structure produit de l'algèbroïde de Hopf $M U_{*}(M U)$, on obtient une action de $\Omega_{*}^{U} \operatorname{sur} \mathcal{E} l l^{*}(M U)=M U_{*}(M U) \otimes_{\Omega^{U}} \mathcal{A}_{*}$ qui lui confère une structure de $\Omega_{*}^{U}$-module. Maintenant, pour tout $\Omega_{*}^{U}$-module $\mathrm{M}$ nous avons :

$$
\begin{aligned}
\operatorname{Tor}_{1, *}^{\Omega_{*}^{U} U}\left(M, \mathcal{E} l l^{*}(M U)\right) & =\operatorname{Tor}_{1, *}^{\Omega_{*}^{U}}\left(M, M U_{*}(M U) \otimes_{\Omega_{*}^{U}} \mathcal{A}_{*}\right) \\
& \simeq \operatorname{Tor}_{1, *}^{\Omega_{*}^{U}}\left(M \otimes_{\Omega_{*} U} M U_{*}(M U), \mathcal{A}_{*}\right)
\end{aligned}
$$

car $M U_{*}(M U)$ est libre (cf. Bourbaki chapitre X, $\$ 6.6$ proposition 8 ). Par ailleurs, $M \otimes_{\Omega_{*}^{U}} M U_{*}(M U)$ est un $M U_{*}(M U)$-comodule, et d'aprés le lemme 3.1, $M \otimes_{\Omega} U M U_{*}(M U)=\operatorname{Lim}_{\alpha} \rightarrow C_{\alpha}$ où $\left\{C_{\alpha}\right\}$ est une famille de $M U_{*}(M U)$-comodules de présentation finie en tant que $\Omega_{*}^{U}$-modules; ceci donne

$$
\begin{gathered}
\operatorname{Tor}_{1, *}^{\Omega_{*}^{U}}\left(M \otimes_{\Omega_{*}^{U}} M U_{*}(M U), \mathcal{A}_{*}\right)=\operatorname{Tor}_{1, *}^{\Omega_{*}^{U}}\left(\operatorname{Lim}_{\alpha} C_{\alpha}, \mathcal{A}_{*}\right) \approx \\
\stackrel{\operatorname{Lim}_{\alpha}}{\longrightarrow} \operatorname{Tor}_{1, *}^{\Omega^{U}}\left(C_{\alpha}, \mathcal{A}_{*}\right) .
\end{gathered}
$$

Mais grâce à ([5], Theorem 3.2) on a $\operatorname{Tor}_{1, *}^{\Omega_{1}^{U}}\left(C_{\alpha}, \mathcal{A}_{*}\right)=0$ pour tout $\alpha$; d'où le résultat.

\section{Théorème 3.3.}

$$
\operatorname{Dimhom}_{\Omega_{*} U}\left(\mathcal{E} l_{*}(M U)\right) \leq 1
$$

Démonstration: $E l l_{*}(M U)$ est un $\Omega_{*}^{U}$-module plat (proposition 3.2). De plus, il est dénombrable en tant que module sur $\Omega_{*}^{U}$ qui est lui même dénombrable donc possède une présentation dénombrable. Le théorème 3.3 découle alors de ([8], Théorème 3.2$)$.

Remarque: $E_{2}^{p, q}=\operatorname{Ext}_{\Omega_{*}^{U}}^{p, q}\left(M U_{*}(E l l), \mathcal{A}_{*}\right)=\operatorname{Ext}_{\Omega_{U} U}^{p, q}\left(E l l_{*}(M U), \mathcal{A}_{*}\right)=0$ pour $p$ différent de 0 et de 1 , grâce au Théorème 3.3 . On retrouve alors la suite exacte scindée (4), c'est à dire :

$0 \rightarrow \operatorname{Ext}_{\Omega_{*}^{U}}^{1, n-1}\left(M U_{*}(E l l), \mathcal{A}_{*}\right) \rightarrow E l l^{n}(E l l) \rightarrow \operatorname{Hom}_{\Omega_{*}^{U}}^{-n}\left(M U_{*}(E l l), \mathcal{A}_{*}\right) \rightarrow 0$ 
Et du fait que $\gamma$ et $\varepsilon$ sont de degré 4 et 8 réspectivement (c.f [6]), $\mathcal{A}_{*}$ est alors $4 \mathbf{Z}$-gradué ; et puisque $\Omega_{*}^{U}$ et $M U_{*}(E l l)$ tant $2 \mathbf{Z}$-gradués on a le

\section{Corollaire 3.4:}

$$
E l l^{n}(E l l)= \begin{cases}E x t_{\Omega^{U}}^{1, n-1}\left(M U_{*}(E l l), \mathcal{A}_{*}\right) & \text { si n est impair } \\ \operatorname{Hom}_{\Omega_{*}^{U}}^{U}\left(M U_{*}(E l l), \mathcal{A}_{*}\right) & \text { si n est pair. }\end{cases}
$$

\section{Références}

[1] J. F. Adams : Stable homotopy and generalised homology. University of Chicago Mathematics Lectures Notes, (1971).

[2] H. Cartan and S. Eilenberg : Homological Algebra. Princeton University Press (sevent printing 1973).

[3] P. E. Conner and E. E. Floyd : The relation of Cobordibm to KTheory. Lecture Notes in Math., No 28 Springer Verlag (1966).

[4] P. E. Conner and L. Smith : On the Complex Bordism of finite Complexes I. I. H. E. S. Pub. Math., No 37 (1969).

[5] P. S. Landweber : Homological Properties of Comodules over MU (MU) and BP (BP). American Journal of Mathematics 98, pp. $591-$ 617 (1976).

[6] P. S. Landweber : Elliptic Cohomology and Modular Forms. Lecture Notes in Math., No 1326 Springer Verlag (1988).

[7] P. S. Landweber, D. C. Ravenel, R. E. Stong : Periodic Cohomology Theories defined by Elliptic Curves. Cechcentenniel conference proceedings, AMS Contempora y Mathematics 181, pp. $317-337$ (1995).

[8] D. Lazar : Autour de la platitude. Bull. Soc. Math. France, No 97, pp. $81-128$ (1969).

[9] H. R. Miller and D. C. Ravenel : Morava Stabilizer Algebra and The Localization of Novikov/s $E_{2}$-term. Ducke Méathematical Journal, 44 (2), pp. $433-447$ (1977). 
Received : October 15, 1995

\section{Abdclaziz Keldouni}

y

Fayçal Lamrini

Département de Mathématique et Informatique

Faculté des Sciences Dhar-Mehraz

Université Sidi Mohammed Benabdallah

B. P. 1796, FES (MAROC)

Marruecos 